\title{
Failure of Cardiovascular Phase 3 Randomized Clinical Trials to Report Pre-trial and Post-trial Parameters: a Cross-sectional Analysis of ClinicalTrials.gov
}

J Gen Intern Med 36(6):1808-10

DOI: $10.1007 / \mathrm{s} 11606-020-05995-9$

(c) Society of General Internal Medicine 2020

\section{INTRODUCTION}

Randomized clinical trials (RCTs) are designed around a small number of pre-specified predicted values, including the trial's hypothesized treatment effect, hypothesized control event rate, alpha, and power, knowing that these values are crucial to understanding trial results and for planning future studies. ${ }^{1,2}$ For example, we would evaluate a null study that failed to meet planned enrollment goals differently than a null study that had a surprisingly low event rate. Here, we assess whether cardiovascular RCTs registered to ClinicalTrials.gov report key predicted values necessary for assessing the intended treatment effect.

\section{METHODS}

ClinicalTrials.gov was queried for all cardiovascular phase 3, randomized, parallel 2-arm RCTs conducted in the USA registered between 2013 and 2014. Information for RCTs came from ClinicalTrials.gov or one of the following: primary publication, protocol publication, or statistical analysis plan provided that the information was directly available from a ClinicalTrials.gov hyperlink. Data extracted included trial status (completed, suspended, terminated, or withdrawn), preresult plans (planned sample size, primary endpoint, alpha, power, statistical plan, hypothesized treatment effect, and hypothesized control group event rate), and post-result data (availability of results on the registry, availability of results in a manuscript, actual sample size, recruitment goal met). All data analyses and visualization were performed in $\mathrm{R}$ version 3.5.3.

\section{RESULTS}

Of the 1011 RCTs registered between 2013 and 2014, there were 373 unique, randomized, parallel 2-arm trials. Of these, 52 were cardiovascular RCTs. All 52 trials included an

Received March 14, 2020

Accepted June 15, 2020

Published online June 24, 2020 estimated sample size, and 33 (63.5\%) of the RCTs met their intended recruitment, as defined by meeting or exceeding the proposed recruitment number (Fig. 1). Of trials that did not reach the intended enrollment, 6 of $52(11.5 \%)$ trials enrolled $75 \%$ or greater of target enrollment, 4 of $52(7.7 \%)$ enrolled between 50 and $75 \%$ of target enrollment, and 9 of 52 (17.3\%) enrolled less than $50 \%$ of target enrollment. Additionally, 17 of $52(32.7 \%)$ failed to reach completion, while $35(67.3 \%)$ were registered as completed. Of cardiovascular RCTs, only 24 of the $52(46.2 \%)$ provided registered results directly available on ClinicalTrials.gov.

Of the 35 completed RCTs, 28 met the pre-specified estimated enrollment $(80.0 \%) ; 22(62.9 \%)$ failed to include at least two of the following pre-specified predicted values: alpha, power, hypothesized treatment effect, and hypothesized control group event rate (Table 1). Only 3 completed RCTs included all 4 of the aforementioned parameters $(8.6 \%)$. Of completed RCTs, 11 (31.4\%) RCTs reported a hypothesized treatment effect, while 5 (14.3\%) trials reported the hypothesized control group event rate. Fewer than half of completed and registered trials $(n=35)$ reported sufficient information to repeat power calculations $(45.8 \%)$.

\section{DISCUSSION}

The pre-specified predicted values that guide a trial are critical to accurately test treatment effect differences. We found suboptimal reporting of RCT study design, making it difficult to assess if cardiovascular trials meet original trial assumptions. Our findings add to the literature revealing the significant underreporting of basic methodological information of RCTs registered on ClinicalTrials.gov.

Prior estimates of reporting and statistical planning of sample sizes of RCTs have largely been based upon published work in major medical journals, and then subsequently looking to trial registries retrospectively, which introduces publication bias. ${ }^{3-5}$ In contrast, this study looks prospectively from registered RCTs, demonstrating far lower rates of reporting of basic required predicted values of RCTs, including the trial's hypothesized treatment effect, hypothesized control group event rate, alpha, and power.

This study is limited by relying upon reporting data from ClinicalTrials.gov, which can have entry errors and could create a possible selection bias around studies that are 


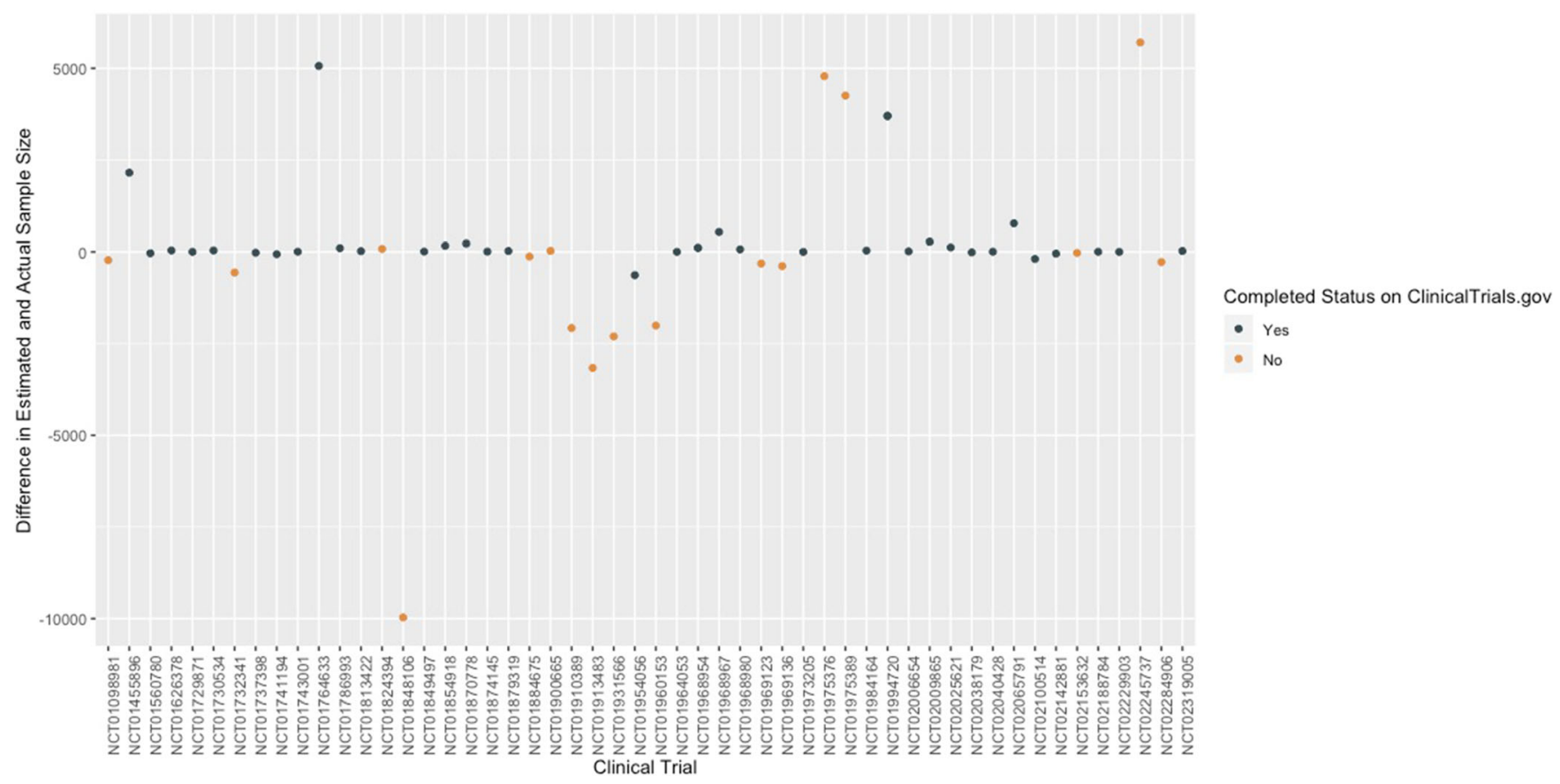

Figure 1 Difference between estimated enrollment and actual enrollment among randomized control trials that were completed versus noncompleted. This figure demonstrates that difference in estimated and actual enrollment by randomized controlled trials related to cardiovascular medicine as registered between 2013 and 2014 and abstracted on February 15, 2019. Randomized controlled trials are further stratified by completion status (completed versus non-completed).

registered and reported. However, ClinicalTrials.gov is meant to be a public repository where investigators can find clinical trial details critical for understanding and replication. Providing trial data and results solely through published manuscripts may limit the availability of trial findings to restricted access journals, and further diminish the utility of ClinicalTrials.gov, a public repository.

Overall, the findings from this study suggest that key predicted values required to power cardiovascular RCTs to meaningfully detect effectiveness of interventions are insufficiently reported at rates greater than previously estimated. Improving pre-trial registration requirements, particularly statistical analysis and participant recruitment planning, as well as post-trial result reporting on a public repository will make ClinicaTtrials.gov more usable and useful while making clinical research more efficient.
Alexander R. Zheutlin, $M D, M S^{1}$

Joshua D. Niforatos, MD, MTS ${ }^{2}$

Eric Stulberg, MD, $M P H^{1}$

Jeremy $B$ Sussman, $M D, M S^{3}$

${ }^{1}$ Department of Internal Medicine, University of Utah, Salt Lake City, UT, USA

${ }^{2}$ Department of Emergency Medicine, The Johns Hopkins Hospital, The Johns Hopkins School of Medicine,

Baltimore, MD, USA

${ }^{3}$ Department of Internal Medicine, Division of General Medicine, University of Michigan,

Ann Arbor, MI, USA

Corresponding Author: Joshua D. Niforatos, MD, MTS; Department of Emergency Medicine, The Johns Hopkins Hospital, The Johns Hopkins School of Medicine Baltimore, MD, USA (e-mail: Jxn187@case.edu).

Table 1 Registered Cardiovascular Clinical Trials and Reporting of Pre-specified Predicted Values

\begin{tabular}{llll}
\hline \hline & $\begin{array}{l}\mathbf{0} \text { or 1 unregistered parameter } \\
(\boldsymbol{n}=\mathbf{1 4})\end{array}$ & $\begin{array}{l}\mathbf{2} \text { or more unregistered parameters } \\
(\boldsymbol{n}=\mathbf{2 3})\end{array}$ & Row totals \\
\hline Results available on ClinicalTrials.gov (row \%) & $12(54.5)$ & $10(45.5)$ & 22 \\
Published manuscript (row \%) & $10(55.0)$ & $8(45.0)$ & 18 \\
Met intended sample size (row \%) & $11(39.3)$ & $7(60.7)$ & 28 \\
Statistical analysis plan included (row \%) & $12(63.2)$ & $8(66.7)$ & 19 \\
Statistically significant results (row \%) & $4(33.3)$ & $18(58.1)$ & 12 \\
Multi-site (row \%) & $13(41.9)$ & 31 \\
\hline
\end{tabular}

This table demonstrates cardiovascular clinical trials that were registered as completed, dichotomized by those that did and did not have two or more of the following pre-specified parameters: alpha, power, hypothesized treatment effect, and hypothesized control group event rate. All data was abstracted on February 15, 2019 
Funding Information This work is supported by VA CDA 13-021 (J.S.).

\section{Compliance with Ethical Standards:}

Conflict of Interest: The authors declare that they do not have a conflict of interest.

\section{REFERENCES}

1. Schulz KF, Grimes DA. Sample size calculations in randomised trials: mandatory and mystical. Lancet. 2005;365(9467):1348-53.

2. Vickers AJ. Underpowering in randomized trials reporting a sample size calculation. J Clin Epidemiol. 2003;56(8):717-20.
3. Naudet F, Sakarovitch C, Janiaud P, Cristea I, Fanelli D, Moher D, Ioannidis JP. Data sharing and reanalysis of randomized controlled trials in leading biomedical journals with a full data sharing policy: survey of studies published in The BMJ and PLOS Medicine. BMJ. 2018;360:k400.

4. Charles P, Giraudeau B, Dechartres A, Baron G, Ravaud P. Reporting of sample size calculation in randomised controlled trials. Bmj. 2009;338:b1732.

5. Zheutlin AR, Niforatos J, Stulberg E, Sussman J. Research Waste in Randomized Clinical Trials: a Cross-Sectional Analysis. J Gen Intern Med. 2019;12:1-3.

Publisher's Note: Springer Nature remains neutral with regard to jurisdictional claims in published maps and institutional affiliations. 